Commun. Korean Math. Soc. 22 (2007), No. 4, pp. 535-545

\title{
ON THE PETTIS INTEGRAL OF FUZZY MAPPINGS IN BANACH SPACES
}

\author{
Chun-Kee Park
}

\begin{abstract}
In this paper, we introduce the Pettis integral of fuzzy mappings in Banach spaces using the Pettis integral of closed set-valued mappings. We investigate the relations between the Pettis integral, weak integral and integral of fuzzy mappings in Banach spaces and obtain some properties of the Pettis integral of fuzzy mappings in Banach spaces.
\end{abstract}

\section{Introduction}

Several types of integrals of set-valued mappings were studied by Amri and Hess [1], Aumann [2], Papageoriou [4], Wu, Zhang and Wang [6] and others. Integrals of fuzzy mappings are generalizations of integrals of set-valued mappings. Kaleva [3] introduced the integral of fuzzy mappings in $\mathbb{R}^{n}$ by use of the integral of set-valued mappings in $\mathbb{R}^{n}$. Xiaoping, Minghu and Ming [7], Xiaoping, Wang and Wu [8] also introduced integrals of fuzzy mappings in Banach spaces by use of Aumann-Pettis and Aumann-Bochner integrals of setvalued mappings. Amri and Hess [1] introduced the Pettis integral of set-valued mappings whose values are closed sets in Banach spaces and established some properties of the integral.

The purpose of this paper is to study the Pettis integral of fuzzy mappings in Banach spaces. We first introduce the Pettis integral of fuzzy mappings in Banach spaces using the Pettis integral of closed set-valued mappings in Banach spaces. And then we investigate the relations between the Pettis integral, weak integral and integral of fuzzy mappings in Banach spaces which were introduced by Xiaoping, Minghu and Ming [7] and obtain some properties of the Pettis integral of fuzzy mappings in Banach spaces.

\section{Preliminaries}

Throughout this paper, $(\Omega, \Sigma, \mu)$ denotes a complete finite measure space and $(X,\|\cdot\|)$ a real separable Banach space with dual $X^{*}$. We write

$P_{0}(X)=\{A: A$ is a nonempty subset of $X\}$,

Received May 1, 2007.

2000 Mathematics Subject Classification. 03E72, 26A39, 28B05, 28B20, 46G10.

Key words and phrases. Pettis integral of closed set-valued mappings, weak integral, integral and Pettis integral of fuzzy mappings in Banach spaces. 
$P_{b(f)(c)}(X)=\left\{A \in P_{0}(X): A\right.$ is bounded (closed)(convex) $\}$,

$P_{w k c}(X)=\left\{A \in P_{0}(X): A\right.$ is weakly compact and convex $\}$.

For $A \subseteq X$ and $x^{*} \in X^{*}$, let $\sigma\left(x^{*}, A\right)=\sup \left\{x^{*}(x): x \in A\right\}$, the support function of $A$.

Let $u: X \rightarrow[0,1]$. We denote $[u]^{r}=\{x \in X: u(x) \geq r\}$ for $r \in(0,1] . u$ is called a generalized fuzzy number if for each $r \in(0,1],[u]^{r} \in P_{w k c}(X)$. Let $\mathcal{F}(X)$ denote the set of all generalized fuzzy numbers on $X$.

For $u, v \in \mathcal{F}(X)$ and $\lambda \in \mathbb{R}$, we define $u+v$ and $\lambda u$ as follows:

$$
\begin{gathered}
(u+v)(x)=\sup _{x=y+z} \min (u(y), v(z)), \\
(\lambda u)(x)= \begin{cases}u\left(\frac{1}{\lambda} x\right), & \lambda \neq 0 \\
\tilde{0}, & \lambda=0, \text { where } \tilde{0}=\chi_{\{0\}} .\end{cases}
\end{gathered}
$$

For $u, v \in \mathcal{F}(X)$ and $\lambda \in \mathbb{R},[u+v]^{r}=[u]^{r}+[v]^{r}$ and $[\lambda u]^{r}=\lambda[u]^{r}$ for each $r \in(0,1]$. Hence $u+v, \lambda u \in \mathcal{F}(X)$.

For $u, v \in \mathcal{F}(X)$, we define $u \leq v$ as follows:

$$
u \leq v \text { if } u(x) \leq v(x) \text { for all } x \in X .
$$

For $u, v \in \mathcal{F}(X), u \leq v$ if and only if $[u]^{r} \subseteq[v]^{r}$ for each $r \in(0,1]$.

For $A, B \in P_{f}(X)$, let $H(A, B)$ denote the Hausdorff metric of $A$ and $B$ defined by

$$
H(A, B)=\max \left(\sup _{a \in A} d(a, B), \sup _{b \in B} d(b, A)\right),
$$

where $d(a, B)=\inf _{b \in B}\|a-b\|$ and $d(b, A)=\inf _{a \in A}\|a-b\|$. Especially,

$$
H(A, B)=\sup _{\left\|x^{*}\right\| \leq 1}\left|\sigma\left(x^{*}, A\right)-\sigma\left(x^{*}, B\right)\right|
$$

whenever $A, B$ are convex sets. Note that $\left(P_{w k c}(X), H\right)$ is a complete metric space. The number $\|A\|$ is defined by

$$
\|A\|=H(A,\{0\})=\sup _{x \in A}\|x\| .
$$

Define $D: \mathcal{F}(X) \times \mathcal{F}(X) \rightarrow[0,+\infty]$ by the equation

$$
D(u, v)=\sup _{r \in(0,1]} H\left([u]^{r},[v]^{r}\right) .
$$

Then $D$ is a metric on $\mathcal{F}(X)$.

The norm $\|u\|$ of $u \in \mathcal{F}(X)$ is defined by

$$
\|u\|=D(u, \tilde{0})=\sup _{r \in(0,1]} H\left([u]^{r},\{0\}\right)=\sup _{r \in(0,1]}\left\|[u]^{r}\right\| .
$$

The mapping $F: \Omega \rightarrow P_{f}(X)$ is called a set-valued mapping. $F$ is said to be scalarly measurable if for every $x^{*} \in X^{*}$, the real-valued function $\sigma\left(x^{*}, F\right)$ is measurable. $F$ is said to be measurable if $F^{-1}(A)=\{\omega \in \Omega: F(\omega) \cap A \neq$ $\emptyset\} \in \Sigma$ for every $A \in P_{f}(X)$. 
Let $F: \Omega \rightarrow P_{f}(X)$. Then the following statements are equivalent [4]:

(1) $F: \Omega \rightarrow P_{f}(X)$ is measurable;

(2) $F^{-1}(U)=\{\omega \in \Omega: F(\omega) \cap U \neq \emptyset\} \in \Sigma$ for every open subset $U$ of $X$;

(3) $\operatorname{Gr}(F)=\{(\omega, x) \in \Omega \times X: x \in F(\omega)\} \in \Sigma \times \mathcal{B}(X)$, where $\mathcal{B}(X)$ is the Borel $\sigma$-algebra of $X$;

(4) (Castaing representation) there exists a sequence $\left\{f_{n}\right\}$ of measurable functions $f_{n}: \Omega \rightarrow X$ such that $F(\omega)=c l\left\{f_{n}(\omega)\right\}$ for all $\omega \in \Omega$.

Note that if $F: \Omega \rightarrow P_{f}(X)$ is measurable then $F: \Omega \rightarrow P_{f}(X)$ is scalarly measurable.

$F: \Omega \rightarrow P_{f}(X)$ is said to be weakly integrably bounded if the real-valued function $\left|x^{*} F\right|: \Omega \rightarrow \mathbb{R},\left|x^{*} F\right|(\omega)=\sup \left\{\left|x^{*}(x)\right|: x \in F(\omega)\right\}$ is integrable for every $x^{*} \in X^{*} . F: \Omega \rightarrow P_{f}(X)$ is said to be integrably bounded if there exists an integrable real-valued function $h$ such that for each $\omega \in \Omega,\|x\| \leq h(\omega)$ for all $x \in F(\omega)$.

$f: \Omega \rightarrow X$ is called a measurable selector of $F: \Omega \rightarrow P_{f}(X)$ if $f$ is measurable and $f(\omega) \in F(\omega)$ for every $\omega \in \Omega$. A measurable selector $f$ of $F$ is called a Pettis (resp., Bochner) integrable selector of $F$ if $f$ is Pettis (resp., Bochner) integrable. We denote by $S_{w F}$ (resp., $S_{F}$ ) the set of all Pettis (resp., Bochner) integrable selectors of $F$.

Given $F: \Omega \rightarrow P_{f}(X)$ and $A \in \Sigma$, the Aumann-Pettis (resp., AumannBochner) integral of $F$ is defined by

$$
\begin{aligned}
& (w) \int_{A} F d \mu=\left\{(P) \int_{A} f d \mu: f \in S_{w F}\right\} \\
& \left(\text { resp., } \int_{A} F d \mu=\left\{\int_{A} f d \mu: f \in S_{F}\right\}\right) .
\end{aligned}
$$

$F: \Omega \rightarrow P_{f}(X)$ is said to be Aumann-Pettis (resp., Aumann-Bochner) integrable if $S_{w F} \neq \emptyset$ (resp., $S_{F} \neq \emptyset$ ).

A measurable extended real-valued function $f$ is said to be quasi-integrable if either $f^{+}$or $f^{-}$is integrable.

$F: \Omega \rightarrow P_{f}(X)$ is said to be scalarly integrable (resp., scalarly quasiintegrable) if for every $x^{*} \in X^{*}, \sigma\left(x^{*}, F\right)$ is integrable (resp., quasi-integrable). $F: \Omega \rightarrow P_{f}(X)$ is said to be scalarly uniformly integrable if the set $\left\{\sigma\left(x^{*}, F\right)\right.$ : $\left.x^{*} \in B_{X^{*}}\right\}$ is uniformly integrable, where $B_{X^{*}}$ is the closed unit ball of $X^{*}$.

A measurable set-valued mapping $F: \Omega \rightarrow P_{f_{c}}(X)$ is said to be Pettis integrable if it satisfies the following two conditions [1]:

(1) $F: \Omega \rightarrow P_{f c}(X)$ is scalarly quasi-integrable,

(2) for every $A \in \Sigma$ there exists $C_{A}(F)=C_{A} \in P_{f c}(X)$ such that

$$
\sigma\left(x^{*}, C_{A}\right)=\int_{A} \sigma\left(x^{*}, F\right) d \mu
$$


for every $x^{*} \in X^{*}$. In this case $C_{A}(F)=(P) \int_{A} F d \mu$ is called the Pettis integral of $F$ over $A$. If $\mathcal{C}$ is a subspace of $P_{f_{c}}(X)$, we say that the set-valued mapping $F: \Omega \rightarrow \mathcal{C}$ is Pettis integrable in $\mathcal{C}$ if $C_{A} \in \mathcal{C}$ for each $A \in \Sigma$.

\section{Results}

A mapping $\tilde{F}: \Omega \rightarrow \mathcal{F}(X)$ is called a fuzzy mapping in a Banach space $X$. In this case $\tilde{F}^{r}: \Omega \rightarrow P_{w k c}(X)$ defined by $\tilde{F}^{r}(\omega)=[\tilde{F}(\omega)]^{r}$ is a set-valued mapping for each $r \in(0,1]$.

A fuzzy mapping $\tilde{F}: \Omega \rightarrow \mathcal{F}(X)$ is said to be measurable if $\tilde{F}^{r}: \Omega \rightarrow$ $P_{w k c}(X)$ is measurable for each $r \in(0,1]$.

Definition 3.1 ([7]). A fuzzy mapping $\tilde{F}: \Omega \rightarrow \mathcal{F}(X)$ is said to be weakly integrable if for each $A \in \Sigma$ there exists $u_{A} \in \mathcal{F}(X)$ such that $\left[u_{A}\right]^{r}=(w) \int_{A} \tilde{F}^{r} d \mu$ for each $r \in(0,1]$. In this case $u_{A}=(w) \int_{A} \tilde{F} d \mu$ is called the weak integral of $\tilde{F}$ over $A$.

A fuzzy mapping $\tilde{F}: \Omega \rightarrow \mathcal{F}(X)$ is said to be integrable if for each $A \in \Sigma$ there exists $u_{A} \in \mathcal{F}(X)$ such that $\left[u_{A}\right]^{r}=\int_{A} \tilde{F}^{r} d \mu$ for each $r \in(0,1]$. In this case $u_{A}=\int_{A} \tilde{F} d \mu$ is called the integral of $\tilde{F}$ over $A$.

Definition 3.2. A measurable fuzzy mapping $\tilde{F}: \Omega \rightarrow \mathcal{F}(X)$ is said to be Pettis integrable if for each $A \in \Sigma$ there exists $u_{A} \in \mathcal{F}(X)$ such that $\left[u_{A}\right]^{r}=$ $(P) \int_{A} \tilde{F}^{r} d \mu$ for each $r \in(0,1]$. In this case $u_{A}=(P) \int_{A} \tilde{F} d \mu$ is called the Pettis integral of $\tilde{F}$ over $A$.

Theorem 3.3. Let $\tilde{F}: \Omega \rightarrow \mathcal{F}(X)$ and $\tilde{G}: \Omega \rightarrow \mathcal{F}(X)$ be Pettis integrable and $\lambda \geq 0$. Then

(1) $\tilde{F}+\tilde{G}$ is Pettis integrable and for each $A \in \Sigma$

$$
(P) \int_{A}(\tilde{F}+\tilde{G}) d \mu=(P) \int_{A} \tilde{F} d \mu+(P) \int_{A} \tilde{G} d \mu,
$$

(2) $\lambda \tilde{F}$ is Pettis integrable and for each $A \in \Sigma$

$$
(P) \int_{A} \lambda \tilde{F} d \mu=\lambda(P) \int_{A} \tilde{F} d \mu \text {. }
$$

Proof. (1) Let $\tilde{F}: \Omega \rightarrow \mathcal{F}(X)$ and $\tilde{G}: \Omega \rightarrow \mathcal{F}(X)$ be Pettis integrable and let $A \in \Sigma$. Then there exist $u_{A}, v_{A} \in \mathcal{F}(X)$ such that $\left[u_{A}\right]^{r}=(P) \int_{A} \tilde{F}^{r} d \mu$, 
$\left[v_{A}\right]^{r}=(P) \int_{A} \tilde{G}^{r} d \mu$ for each $r \in(0,1]$. Therefore

$$
\sigma\left(x^{*},\left[u_{A}\right]^{r}\right)=\int_{A} \sigma\left(x^{*}, \tilde{F}^{r}\right) d \mu, \quad \sigma\left(x^{*},\left[v_{A}\right]^{r}\right)=\int_{A} \sigma\left(x^{*}, \tilde{G}^{r}\right) d \mu
$$

for each $r \in(0,1]$ and $x^{*} \in X^{*}$. Hence we have

$$
\begin{aligned}
\sigma\left(x^{*},\left[u_{A}+v_{A}\right]^{r}\right) & =\sigma\left(x^{*},\left[u_{A}\right]^{r}+\left[v_{A}\right]^{r}\right) \\
& =\sigma\left(x^{*},\left[u_{A}\right]^{r}\right)+\sigma\left(x^{*},\left[v_{A}\right]^{r}\right) \\
& =\int_{A} \sigma\left(x^{*}, \tilde{F}^{r}\right) d u+\int_{A} \sigma\left(x^{*}, \tilde{G}^{r}\right) d \mu \\
& =\int_{A}\left(\sigma\left(x^{*}, \tilde{F}^{r}\right)+\sigma\left(x^{*}, \tilde{G}^{r}\right)\right) d \mu \\
& =\int_{A} \sigma\left(x^{*}, \tilde{F}^{r}+\tilde{G}^{r}\right) d \mu \\
& =\int_{A} \sigma\left(x^{*},(\tilde{F}+\tilde{G})^{r}\right) d \mu
\end{aligned}
$$

for each $r \in(0,1]$ and $x^{*} \in X^{*}$. Thus $\left[u_{A}+v_{A}\right]^{r}=(P) \int_{A}(\tilde{F}+\tilde{G})^{r} d \mu$ for each $r \in(0,1]$. Hence $\tilde{F}+\tilde{G}$ is Pettis integrable and for each $A \in \Sigma$

$$
(P) \int_{A}(\tilde{F}+\tilde{G}) d \mu=u_{A}+v_{A}=(P) \int_{A} \tilde{F} d \mu+(P) \int_{A} \tilde{G} d \mu .
$$

(2) Let $\tilde{F}: \Omega \rightarrow \mathcal{F}(X)$ be Pettis integrable and $\lambda \geq 0$. Then for each $A \in \Sigma$ there exists $u_{A} \in \mathcal{F}(X)$ such that $\left[u_{A}\right]^{r}=(P) \int_{A} \tilde{F}^{r} d \mu$ for each $r \in(0,1]$. Since $\sigma\left(x^{*},\left[\lambda u_{A}\right]^{r}\right)=\sigma\left(x^{*}, \lambda\left[u_{A}\right]^{r}\right)=\lambda \sigma\left(x^{*},\left[u_{A}\right]^{r}\right)$ for each $r \in(0,1]$ and $x^{*} \in X^{*}$, using the same method as (1) we obtain that $\lambda \tilde{F}$ is Pettis integrable and for each $A \in \Sigma$

$$
\text { (P) } \int_{A} \lambda \tilde{F} d \mu=\lambda(P) \int_{A} \tilde{F} d \mu
$$

Theorem 3.4. Let $\tilde{F}: \Omega \rightarrow \mathcal{F}(X)$ and $\tilde{G}: \Omega \rightarrow \mathcal{F}(X)$ be Pettis integrable. Then

(1) if $\tilde{F}(\omega) \leq \tilde{G}(\omega) \mu$-a.e., then $(P) \int_{A} \tilde{F} d \mu \leq(P) \int_{A} \tilde{G} d \mu$ for each $A \in \Sigma$;

(2) if $\tilde{F}(\omega)=\tilde{G}(\omega) \mu$-a.e., then $(P) \int_{A} \tilde{F} d \mu=(P) \int_{A}^{A} \tilde{G} d \mu$ for each $A \in \Sigma$.

Proof. (1) Since $\tilde{F}: \Omega \rightarrow \mathcal{F}(X)$ and $\tilde{G}: \Omega \rightarrow \mathcal{F}(X)$ are Pettis integrable, for each $A \in \Sigma$ there exist $u_{A}, v_{A} \in \mathcal{F}(X)$ such that $u_{A}=(P) \int_{A} \tilde{F} d \mu, v_{A}=$ (P) $\int_{A} \tilde{G} d \mu$. If $\tilde{F}(\omega) \leq \tilde{G}(\omega) \mu$-a.e., then $\tilde{F}^{r}(\omega) \leq \tilde{G}^{r}(\omega) \mu$-a.e. for each 
$r \in(0,1]$. By $\left[1\right.$, Proposition 4.1], $\left[u_{A}\right]^{r}=(P) \int_{A} \tilde{F}^{r} d \mu \subseteq(P) \int_{A} \tilde{G}^{r} d \mu=\left[v_{A}\right]^{r}$ for each $r \in(0,1]$ and $A \in \Sigma$. Thus $(P) \int_{A} \tilde{F} d \mu \leq(P) \int_{A} \tilde{G} d \mu$ for each $A \in \Sigma$.

(2) The proof is similar to (1).

A fuzzy mapping $\tilde{F}: \Omega \rightarrow \mathcal{F}(X)$ is said to be weakly integrably bounded if $\tilde{F}^{r}: \Omega \rightarrow P_{w k c}(X)$ is weakly integrably bounded for each $r \in(0,1][5]$.

A fuzzy mapping $\tilde{F}: \Omega \rightarrow \mathcal{F}(X)$ is said to be scalarly integrable (resp., scalarly uniformly integrable) if $\tilde{F}^{r}: \Omega \rightarrow P_{w k c}(X)$ is scalarly integrable (resp., scalarly uniformly integrable) for each $r \in(0,1]$.

Theorem 3.5. If $\tilde{F}: \Omega \rightarrow \mathcal{F}(X)$ is measurable, weakly integrably bounded and scalarly uniformly integrable, then $\tilde{F}: \Omega \rightarrow \mathcal{F}(X)$ is Pettis integrable.

Proof. Let $A \in \Sigma$. Since $\tilde{F}: \Omega \rightarrow \mathcal{F}(X)$ is measurable and scalarly uniformly integrable, by [1, Theorem 5.4] $\tilde{F}^{r}: \Omega \rightarrow P_{w k c}(X)$ is Pettis integrable in $P_{w k c}(X)$ for each $r \in(0,1]$. Thus $M_{r}=(P) \int_{A} \tilde{F}^{r} d \mu \in P_{w k c}(X)$ for each $r \in(0,1]$. For $r_{1}, r_{2} \in(0,1]$ with $r_{1}<r_{2}, \tilde{F}^{r_{1}}(\omega) \supseteq \tilde{F}^{r_{2}}(\omega)$ for each $\omega \in \Omega$. By [1, Proposition 4.1] $M_{r_{1}}=(P) \int_{A} \tilde{F}^{r_{1}} d \mu \supseteq(P) \int_{A} \tilde{F}^{r_{2}} d \mu=M_{r_{2}}$. Let $r \in(0,1]$ and $\left\{r_{n}\right\}$ be a sequence in $(0,1]$ such that $r_{1} \leq r_{2} \leq r_{3} \leq \cdots$ and $\lim _{n \rightarrow \infty} r_{n}=r$. Then $\tilde{F}^{r}(\omega)=\cap_{n=1}^{\infty} \tilde{F}^{r_{n}}(\omega)$ for each $\omega \in \Omega$. By [7, Lemma 4.2] $\lim _{n \rightarrow \infty} \sigma\left(x^{*}, \tilde{F}^{r_{n}}(\omega)\right)=\sigma\left(x^{*}, \tilde{F}^{r}(\omega)\right)$ for each $\omega \in \Omega$ and $x^{*} \in X^{*}$. For each $n \in \mathbb{N},\left|\sigma\left(x^{*}, \tilde{F}^{r_{n}}(\omega)\right)\right| \leq\left|x^{*} \tilde{F}^{r_{1}}\right|(\omega)$ for each $\omega \in \Omega$ and $x^{*} \in X^{*}$. Since $\tilde{F}: \Omega \rightarrow \mathcal{F}(X)$ is weakly integrably bounded, by Lebesgue dominated convergence theorem we have

$$
\lim _{n \rightarrow \infty} \sigma\left(x^{*}, M_{r_{n}}\right)=\lim _{n \rightarrow \infty} \int_{A} \sigma\left(x^{*}, \tilde{F}^{r_{n}}\right) d \mu=\int_{A} \sigma\left(x^{*}, \tilde{F}^{r}\right) d \mu=\sigma\left(x^{*}, M_{r}\right)
$$

for each $x^{*} \in X^{*}$. By [7, Lemma 4.2] $M_{r}=\cap_{n=1}^{\infty} M_{r_{n}}$. Let $M_{0}=X$. Then by $\left[7\right.$, Lemma 4.1] there exists $u_{A} \in \mathcal{F}(X)$ such that $\left[u_{A}\right]^{r}=M_{r}=(P) \int_{A} \tilde{F}^{r} d \mu$ for each $r \in(0,1]$. Hence $\tilde{F}: \Omega \rightarrow \mathcal{F}(X)$ is Pettis integrable.

Theorem 3.6. If $\tilde{F}: \Omega \rightarrow \mathcal{F}(X)$ is scalarly integrable and Pettis integrable, then $\tilde{F}: \Omega \rightarrow \mathcal{F}(X)$ is scalarly uniformly integrable.

Proof. If $\tilde{F}: \Omega \rightarrow \mathcal{F}(X)$ is scalarly integrable and Pettis integrable, then for each $r \in(0,1] \tilde{F}^{r}: \Omega \rightarrow P_{w k c}(X)$ is measurable and scalarly integrable and for each $A \in \Sigma$ there exists $u_{A} \in \mathcal{F}(X)$ such that $\left[u_{A}\right]^{r}=(P) \int_{A} \tilde{F}^{r} d \mu$ for each $r \in(0,1]$. Thus $\tilde{F}^{r}: \Omega \rightarrow P_{w k c}(X)$ is Pettis integrable for each $r \in(0,1]$. By 
[1, Theorem 5.4] $\tilde{F}^{r}: \Omega \rightarrow P_{w k c}(X)$ is scalarly uniformly integrable for each $r \in(0,1]$. Hence $\tilde{F}: \Omega \rightarrow \mathcal{F}(X)$ is scalarly uniformly integrable.

Remark 3.7. If $\tilde{F}: \Omega \rightarrow \mathcal{F}(X)$ is weakly integrably bounded, then $\tilde{F}: \Omega \rightarrow$ $\mathcal{F}(X)$ is scalarly integrable. But the converse is not true.

We can obtain the following corollary from Theorem 3.5, Theorem 3.6 and Remark 3.7.

Corollary 3.8. Let $\tilde{F}: \Omega \rightarrow \mathcal{F}(X)$ be measurable and weakly integrably bounded. Then $\tilde{F}: \Omega \rightarrow \mathcal{F}(X)$ is Pettis integrable if and only if $\tilde{F}: \Omega \rightarrow \mathcal{F}(X)$ is scalarly uniformly integrable.

$\tilde{F}: \Omega \rightarrow \mathcal{F}(X)$ is said to be integrably bounded if there exists an integrable real-valued function $h$ such that for each $\omega \in \Omega,\|x\| \leq h(\omega)$ for all $x \in \tilde{F}^{0}(\omega)$, where $\tilde{F}^{0}(\omega)=c l\left(\cup_{0<r \leq 1} \tilde{F}^{r}(\omega)\right)$. If $\tilde{F}: \Omega \rightarrow \mathcal{F}(X)$ is integrably bounded, then $\tilde{F}: \Omega \rightarrow \mathcal{F}(X)$ is weakly integrably bounded.

Lemma 3.9. If $F: \Omega \rightarrow P_{w k c}(X)$ and $G: \Omega \rightarrow P_{w k c}(X)$ are integrably bounded and Pettis integrable in $P_{w k c}(X)$, then $H(F, G)$ is integrable and

$$
H\left((P) \int_{\Omega} F d \mu,(P) \int_{\Omega} G d \mu\right) \leq \int_{\Omega} H(F, G) d \mu .
$$

Proof. Since $F$ and $G$ are measurable, there exist Castaing representations $\left\{f_{n}\right\}$ and $\left\{g_{n}\right\}$ for $F$ and $G$. Since $f_{n}$ and $g_{n}$ are measurable for all $n \in \mathbb{N}$,

$$
H(F(\omega), G(\omega))=\max \left(\sup _{n \geq 1} \inf _{k \geq 1}\left\|f_{n}(\omega)-g_{k}(\omega)\right\|, \sup _{n \geq 1} \inf _{k \geq 1}\left\|g_{n}(\omega)-f_{k}(\omega)\right\|\right)
$$

is measurable. Since $F$ and $G$ are integrably bounded, there exist integrable real-valued functions $h_{1}$ and $h_{2}$ such that for each $\omega \in \Omega,\|x\| \leq h_{1}(\omega)$ for all $x \in F(\omega)$ and $\|x\| \leq h_{2}(\omega)$ for all $x \in G(\omega)$. Hence we have

$$
H(F(\omega), G(\omega)) \leq H(F(\omega),\{0\})+H(G(\omega),\{0\}) \leq h_{1}(\omega)+h_{2}(\omega)
$$

for each $\omega \in \Omega$. Therefore $H(F, G)$ is integrable and by [1, Proposition 2.2] we have

$$
\begin{aligned}
& H\left((P) \int_{\Omega} F d \mu,(P) \int_{\Omega} G d \mu\right) \\
= & \sup _{\left\|\boldsymbol{x}^{*}\right\| \leq 1}\left|\sigma\left(x^{*},(P) \int_{\Omega} F d \mu\right)-\sigma\left(x^{*},(P) \int_{\Omega} G d \mu\right)\right|
\end{aligned}
$$




$$
\begin{aligned}
& =\sup _{\left\|x^{*}\right\| \leq 1}\left|\int_{\Omega} \sigma\left(x^{*}, F\right) d \mu-\int_{\Omega} \sigma\left(x^{*}, G\right) d \mu\right| \\
& \leq \sup _{\left\|x^{*}\right\| \leq 1} \int_{\Omega}\left|\sigma\left(x^{*}, F\right)-\sigma\left(x^{*}, G\right)\right| d \mu \\
& \leq \int_{\Omega} \sup _{\left\|x^{*}\right\| \leq 1}\left|\sigma\left(x^{*}, F\right)-\sigma\left(x^{*}, G\right)\right| d \mu \\
& =\int_{\Omega} H(F, G) d \mu .
\end{aligned}
$$

Theorem 3.10. If $\tilde{F}: \Omega \rightarrow \mathcal{F}(X)$ and $\tilde{G}: \Omega \rightarrow \mathcal{F}(X)$ are integrably bounded and Pettis integrable, then $D(\tilde{F}, \tilde{G})$ is integrable and

$$
D\left((P) \int_{\Omega} \tilde{F} d \mu,(P) \int_{\Omega} \tilde{G} d \mu\right) \leq \int_{\Omega} D(\tilde{F}, \tilde{G}) d \mu .
$$

Proof. Since $\tilde{F}$ and $\tilde{G}$ are measurable, there exist Castaing representations $\left\{f_{n}^{r}\right\}$ and $\left\{g_{n}^{r}\right\}$ for $\tilde{F}^{r}$ and $\tilde{G}^{r}$ for each $r \in(0,1]$. Since $f_{n}^{r}$ and $g_{n}^{r}$ are measurable for all $n \in \mathbb{N}$,

$$
H\left(\tilde{F}^{r}(\omega), \tilde{G}^{r}(\omega)\right)=\max \left(\sup _{n \geq 1} \inf _{k \geq 1}\left\|f_{n}^{r}(\omega)-g_{k}^{r}(\omega)\right\|, \sup _{n \geq 1} \inf _{k \geq 1}\left\|g_{n}^{r}(\omega)-f_{k}^{r}(\omega)\right\|\right)
$$

is measurable for each $r \in(0,1]$. Hence

$$
D(\tilde{F}(\omega), \tilde{G}(\omega))=\sup _{k \geq 1} H\left(\tilde{F}^{r_{k}}(\omega), \tilde{G}^{r_{k}}(\omega)\right)
$$

is measurable, where $\left\{r_{k}: k \in \mathbb{N}\right\}$ is dense in $(0,1]$. Since $\tilde{F}$ and $\tilde{G}$ are integrably bounded, there exist integrable real-valued functions $h_{1}$ and $h_{2}$ such that for each $\omega \in \Omega,\|x\| \leq h_{1}(\omega)$ for all $x \in \tilde{F}^{0}(\omega)$ and $\|x\| \leq h_{2}(\omega)$ for all $x \in \tilde{G}^{0}(\omega)$. Hence we have

$$
D(\tilde{F}(\omega), \tilde{G}(\omega)) \leq D(\tilde{F}(\omega), \tilde{0})+D(\tilde{G}(\omega), \tilde{0}) \leq h_{1}(\omega)+h_{2}(\omega)
$$

for each $\omega \in \Omega$. Therefore $D(\tilde{F}, \tilde{G})$ is integrable and by Lemma 3.9

$$
H\left((P) \int_{\Omega} \tilde{F}^{r} d \mu,(P) \int_{\Omega} \tilde{G}^{r} d \mu\right) \leq \int_{\Omega} H\left(\tilde{F}^{r}, \tilde{G}^{r}\right) d \mu
$$

for each $r \in(0,1]$. Hence we have

$$
\begin{aligned}
& D\left((P) \int_{\Omega} \tilde{F} d \mu,(P) \int_{\Omega} \tilde{G} d \mu\right) \\
= & \sup _{r \in(0,1]} H\left(\left[(P) \int_{\Omega} \tilde{F} d \mu\right]^{r},\left[(P) \int_{\Omega} \tilde{G} d \mu\right]^{r}\right)
\end{aligned}
$$




$$
\begin{aligned}
& =\sup _{r \in(0,1]} H\left((P) \int_{\Omega} \tilde{F}^{r} d \mu,(P) \int_{\Omega} \tilde{G}^{r} d \mu\right) \\
& \leq \sup _{r \in(0,1]} \int_{\Omega} H\left(\tilde{F}^{r}, \tilde{G}^{r}\right) d \mu \\
& \leq \int_{\Omega} \sup _{r \in(0,1]} H\left(\tilde{F}^{r}, \tilde{G}^{r}\right) d \mu \\
& =\int_{\Omega} D(\tilde{F}, \tilde{G}) d \mu .
\end{aligned}
$$

Theorem 3.11. If a measurable fuzzy mapping $\tilde{F}: \Omega \rightarrow \mathcal{F}(X)$ is integrable, then $\tilde{F}: \Omega \rightarrow \mathcal{F}(X)$ is weakly integrable and $\int_{A} \tilde{F} d \mu=(w) \int_{A} \tilde{F} d \mu$ for each $A \in \Sigma$.

Proof. If $\tilde{F}: \Omega \rightarrow \mathcal{F}(X)$ is integrable, then for each $A \in \Sigma$ there exists $u_{A} \in$ $\mathcal{F}(X)$ such that $u_{A}=\int_{A} \tilde{F} d \mu$. Thus $\left[u_{A}\right]^{r}=\int_{A} \tilde{F}^{r} d \mu$ for each $r \in(0,1]$. Since $\tilde{F}^{r}: \Omega \rightarrow P_{w k c}(X)$ is Aumann-Bochner integrable for each $r \in(0,1], \tilde{F}^{r}: \Omega \rightarrow$ $P_{w k c}(X)$ is Aumann-Pettis integrable and $w$-cl $\int_{A} \tilde{F}^{r} d \mu=w-c l(w) \int_{A} \tilde{F}^{r} d \mu$ for each $r \in(0,1]$ by $\left[1\right.$, Proposition 3.12]. Since $\left[u_{A}\right]^{r}=\int_{A} \tilde{F}^{r} d \mu \in P_{w k c}(X)$ for each $r \in(0,1], \int_{A} \tilde{F}^{r} d \mu=w-c l \int_{A} \tilde{F}^{r} d \mu=w-c l(w) \int_{A} \tilde{F}^{r} d \mu \supseteq(w) \int_{A} \tilde{F}^{r} d \mu$ for each $r \in(0,1]$. Generally, $\int_{A} \tilde{F}^{r} d \mu \subseteq(w) \int_{A} \tilde{F}^{r} d \mu$ for each $r \in(0,1]$. Hence $\left[u_{A}\right]^{r}=\int_{A} \tilde{F}^{r} d \mu=(w) \int_{A} \tilde{F}^{r} d \mu$ for each $r \in(0,1]$. Thus $u_{A}=(w) \int_{A} \tilde{F} d \mu$. Therefore $\tilde{F}: \Omega \rightarrow \mathcal{F}(X)$ is weakly integrable and $\int_{A} \tilde{F} d \mu=(w) \int_{A} \tilde{F} d \mu$ for each $A \in \Sigma$.

Theorem 3.12. If a measurable fuzzy mapping $\tilde{F}: \Omega \rightarrow \mathcal{F}(X)$ is weakly integrable, then $\tilde{F}: \Omega \rightarrow \mathcal{F}(X)$ is Pettis integrable and $(w) \int_{A} \tilde{F} d \mu=(P) \int_{A} \tilde{F} d \mu$ for each $A \in \Sigma$.

Proof. If $\tilde{F}: \Omega \rightarrow \mathcal{F}(X)$ is weakly integrable, then for each $A \in \Sigma$ there exists $u_{A} \in \mathcal{F}(X)$ such that $u_{A}=(w) \int_{A} \tilde{F} d \mu$. Thus $\left[u_{A}\right]^{r}=(w) \int_{A} \tilde{F}^{r} d \mu$ for each $r \in(0,1]$. Since $\tilde{F}^{r}: \Omega \rightarrow P_{w k c}(X)$ is measurable and Aumann-Pettis integrable for each $r \in(0,1]$, by [1, Theorem 3.7] $\tilde{F}^{r}: \Omega \rightarrow P_{w k c}(X)$ is Pettis integrable for each $r \in(0,1]$ and by $\left[7\right.$, Lemma 4.3] $\sigma\left(x^{*},(w) \int_{A} \tilde{F}^{r} d \mu\right)=$ 
$\int_{A} \sigma\left(x^{*}, \tilde{F}^{r}\right) d \mu$ for each $x^{*} \in X^{*}$ and $r \in(0,1]$. Hence $\left[u_{A}\right]^{r}=(w) \int_{A} \tilde{F}^{r} d \mu$ $=(P) \int_{A} \tilde{F}^{r} d \mu$ for each $r \in(0,1]$. Thus $u_{A}=(P) \int_{A} \tilde{F} d \mu$. Therefore $\tilde{F}: \Omega \rightarrow$ $\mathcal{F}(X)$ is Pettis integrable and $(w) \int_{A} \tilde{F} d \mu=(P) \int_{A} \tilde{F} d \mu$ for each $A \in \Sigma$.

We can obtain the following two corollaries from Theorem 3.10, Theorem 3.11 and Theorem 3.12

Corollary 3.13. If measurable fuzzy mappings $\tilde{F}: \Omega \rightarrow \mathcal{F}(X)$ and $\tilde{G}: \Omega \rightarrow$ $\mathcal{F}(X)$ are integrably bounded and integrable, then $D(\tilde{F}, \tilde{G})$ is integrable and

$$
D\left(\int_{\Omega} \tilde{F} d \mu, \int_{\Omega} \tilde{G} d \mu\right) \leq \int_{\Omega} D(\tilde{F}, \tilde{G}) d \mu .
$$

Corollary 3.14. If measurable fuzzy mappings $\tilde{F}: \Omega \rightarrow \mathcal{F}(X)$ and $\tilde{G}: \Omega \rightarrow$ $\mathcal{F}(X)$ are integrably bounded and weakly integrable, then $D(\tilde{F}, \tilde{G})$ is integrable and

$$
D\left((w) \int_{\Omega} \tilde{F} d \mu,(w) \int_{\Omega} \tilde{G} d \mu\right) \leq \int_{\Omega} D(\tilde{F}, \tilde{G}) d \mu .
$$

Theorem 3.15. Let $X$ contain no copy of $c_{0}$ and let $\tilde{F}_{n}: \Omega \rightarrow \mathcal{F}(X)(n \in \mathbb{N})$ and $\tilde{F}: \Omega \rightarrow \mathcal{F}(X)$ be measurable fuzzy mappings such that

$$
\lim _{n \rightarrow \infty} D\left(\tilde{F}_{n}(\omega), \tilde{F}(\omega)\right)=0
$$

on $\Omega$. If there exists an integrable real-valued function $h$ such that $\left\|\tilde{F}_{n}^{0}(\omega)\right\| \leq$ $h(\omega)$ on $\Omega$ for each $n \in \mathbb{N}$, then $\tilde{F}: \Omega \rightarrow \mathcal{F}(X)$ is weakly integrable and

$$
\lim _{n \rightarrow \infty} D\left((w) \int_{\Omega} \tilde{F}_{n} d \mu,(w) \int_{\Omega} \tilde{F} d \mu\right)=0 .
$$

Proof. Since $\lim _{n \rightarrow \infty} D\left(\tilde{F}_{n}(\omega), \tilde{F}(\omega)\right)=0$ on $\Omega$, for each $\epsilon>0$ and $\omega \in \Omega$ there exists $N \in \mathbb{N}$ such that $n \geq N \Rightarrow D\left(\tilde{F}_{n}(\omega), \tilde{F}(\omega)\right)<\epsilon$. For some $n \in \mathbb{N}$ with $n \geq N$,

$$
\begin{aligned}
\left\|\tilde{F}^{0}(\omega)\right\| & =D(\tilde{F}(\omega), \tilde{0}) \leq D\left(\tilde{F}(\omega), \tilde{F}_{n}(\omega)\right)+D\left(\tilde{F}_{n}(\omega), \tilde{0}\right) \\
& <\left\|\tilde{F}_{n}^{0}(\omega)\right\|+\epsilon \leq h(\omega)+\epsilon .
\end{aligned}
$$

Since $\epsilon>0$ is arbitrary, $\left\|\tilde{F}^{0}(\omega)\right\| \leq h(\omega)$ on $\Omega$. Thus $\tilde{F}_{n}: \Omega \rightarrow \mathcal{F}(X)(n \in \mathbb{N})$ and $\tilde{F}: \Omega \rightarrow \mathcal{F}(X)$ are integrably bounded and so weakly integrably bounded. By [7, Theorem 4.5], $\tilde{F}_{n}: \Omega \rightarrow \mathcal{F}(X)(n \in \mathbb{N})$ and $\tilde{F}: \Omega \rightarrow \mathcal{F}(X)$ are weakly integrable. By Corollary 3.14 and Lebesgue dominated convergence theorem,

$$
D\left((w) \int_{\Omega} \tilde{F}_{n} d \mu,(w) \int_{\Omega} \tilde{F} d \mu\right) \leq \int_{\Omega} D\left(\tilde{F}_{n}, \tilde{F}\right) d \mu \rightarrow 0 \text { as } n \rightarrow \infty .
$$

Thus $\lim _{n \rightarrow \infty} D\left((w) \int_{\Omega} \tilde{F}_{n} d \mu,(w) \int_{\Omega} \tilde{F} d \mu\right)=0$. 
We can obtain the following corollary from Theorem 3.12 and Theorem 3.15.

Corollary 3.16. Let $X$ contain no copy of $c_{0}$ and let $\tilde{F}_{n}: \Omega \rightarrow \mathcal{F}(X)(n \in \mathbb{N})$ and $\tilde{F}: \Omega \rightarrow \mathcal{F}(X)$ be measurable fuzzy mappings such that

$$
\lim _{n \rightarrow \infty} D\left(\tilde{F}_{n}(\omega), \tilde{F}(\omega)\right)=0
$$

on $\Omega$. If there exists an integrable real-valued function $h$ such that $\left\|\tilde{F}_{n}^{0}(\omega)\right\| \leq$ $h(\omega)$ on $\Omega$ for each $n \in \mathbb{N}$, then $\tilde{F}: \Omega \rightarrow \mathcal{F}(X)$ is Pettis integrable and

$$
\lim _{n \rightarrow \infty} D\left((P) \int_{\Omega} \tilde{F}_{n} d \mu,(P) \int_{\Omega} \tilde{F} d \mu\right)=0 .
$$

\section{References}

[1] K. Amri and C. Hess, On the Pettis integral of closed valued multifunctions, Set-Valued Analysis 8 (2000), 329-360.

[2] R. J. Aumann, Integrals of set-valued functions, J. Math. Anal. Appl. 12 (1965), 1-12.

[3] O. Kaleva, Fuzzy differential equations, Fuzzy Sets and Systems 24 (1987), 301-317.

[4] N. Papageoriou, On the theory of Banach space valued multifunctions, J. Multivariate Anal. 17 (1985), 185-206.

[5] J. Wu and C. Wu, The w-derivatives of fuzzy mappings in Banach spaces, Fuzzy Sets and Systems 119 (2001), 375-381.

[6] W. Z. Wu, W. X. Zhang, and R. M. Wang, Set valued Bartle integrals, J. Math. Anal. Appl. 255 (2001), 1-20.

[7] X. Xiaoping, H. Minghu, and M. Ming, Random fuzzy number integrals in Banach spaces, Fuzzy Sets and Systems 66 (1994), 97-111.

[8] X. Xiaoping, X. Wang, and $\mathrm{L}$. Wu On the convergence and representation of random fuzzy number integrals, Fuzzy Sets and Systems 103 (1999), 115-125.

Department of Mathematics

KaNGWON NATIONAL UNIVERSity

Chuncheon 200-701, Korea

E-mail address: ckpark@kangwon.ac.kr 